\title{
THEMATIC \\ Perinatal mental health around the world: priorities for research and service development in Sweden
}

\author{
Birgitta Wickberg, ${ }^{1} \odot$ Marie Bendix, ${ }^{2} \odot$ Margareta Blomdahl Wetterholm ${ }^{2}$ \\ and Alkistis Skalkidou ${ }^{3}$
}

\begin{abstract}
Associate Professor, Department of Psychology, University of Gothenburg, Göteborg, Sweden. Email: birgitta.wickberg@gmail. com

${ }^{2}$ Liaison Perinatal Consultant Psychiatrist, Centre for Psychiatry Research \& Stockholm Health Care Services, Stockholm County Counc olinska Institutet Stockholm, Sweden

${ }^{3}$ Professor, Department of Women's and Children's Health, Uppsala University, Sweden
\end{abstract}

Conflicts of interest: None.

Keywords. Universal screening; multidisciplinary teams;

immigrants.

First received 16 May 2019 Final revision 13 Jul 2019 Accepted 27 Jul 2019

doi:10.1192/bji.2019.23

(c) The Authors 2019. This is an Open Access article, distributed under the terms of the Creative Commons Attribution licence (http://creativecommons.org/ licenses/by/4.0/), which permits (1) and reproduction in any medium, provided the original work is properly cited.

\begin{abstract}
Sweden has a unique opportunity to identify and follow up women presenting with, or at risk for, perinatal mental health problems and disorders because universal screening programmes are provided by its primary healthcare system. Although they are implemented across almost the entire population, screening programmes are not necessarily leading to effective interventions because the multidisciplinary perinatal mental healthcare teams that provide for the assessment and treatment of moderate to severe disorders are very few in number and must be increased. In particular, efforts to reach immigrant parents must be intensified to achieve equal quality of care for all.
\end{abstract}

Sweden, a Nordic country with ten million inhabitants, has in one generation changed from an ethnically homogeneous country to one of the most heterogeneous in Europe. Twenty-four per cent of the population is of foreign origin, either born abroad or having both parents born abroad. ${ }^{1}$ Whatever their reason for migrating to Sweden, all immigrant groups report having poorer health, including mental health, than the rest of the population. ${ }^{2}$ Over the past decade, mental health problems have increased, and psychiatric diagnoses are now the most common reason for sick leave, the proportion having more than doubled between 2010 and 2015. Adjustment disorders and other reactions to severe stress account for almost two-thirds of the total increase in sick leave, especially among people in younger middle age. ${ }^{3}$ There are many possible factors contributing to this increase, such as the stress inherent in trying to balance the demands of work and family life. One way to identify mental health needs in the population as a whole is to focus on the childbearing period. At this time, people are usually open to lifestyle changes and to receiving care, while they are in very close contact with the healthcare system. This offers an optimal opportunity for primary prevention and early interventions.

\section{Antenatal and child healthcare services}

In Sweden, the antenatal and Child Health Services (CHS) have a long tradition, are free of charge and almost $100 \%$ of pregnant women and new parents with infants attend regular appointments. Antenatal care is mainly delivered by midwives and offers 8-11 recommended check-ups during pregnancy, and one postpartum appointment. The child healthcare programme is offered to all children between 0 and 5 years as well as parents, regardless of income or risk exposure. In recent decades there has been a shift from what used to be primarily somatic management concerns about pregnancy and postnatal care towards an increasingly public health and psychosocial perspective. The CHS team is led by a nurse, and includes a general practitioner or a paediatrician and, in almost all counties of Sweden, a psychologist. These psychologists work within the antenatal and child healthcare systems, offering regular consultation meetings with midwives and nurses and providing easy access for assessment and treatment to women with perinatal mental health problems.

\section{Early identification}

Much research in perinatal mental health has focused on postnatal depression, but in the past few years this perspective has broadened to include mental illness during pregnancy and a wide range of other mental disorders. Programmes that combine the systematic identification of postnatal depression with enhanced support have proven to be both clinically valuable and cost-effective (for a review see Howard $e t a l^{4}$ ). Whether universal prenatal screening for depression is clinically effective and cost-effective is still under discussion. Some counties in Sweden are nevertheless implementing pilot projects. Antenatal screening for depression and other current or past mental health problems is by questionnaires or structured interviews. ${ }^{5}$ One county recently introduced a telephone call, 2 weeks after delivery, to ask about the mother's mental state. The effectiveness of these approaches in improving postnatal health is not yet established.

In 2010, the Swedish National Board of Health and Welfare recommended postnatal screening for depression using the Edinburgh Postnatal Depression Scale (EPDS), to be followed by person-centred counselling (so-called 'listening visits') by CHS nurses for all women with subthreshold or minor depression. This procedure is now implemented all over the country. ${ }^{6}$ 
National figures on postnatal screening rates are not available but in the three largest regions of Sweden, which include more than half of the population, about four out of five women took part in the screening visit $6-8$ weeks postpartum during 2017. In the southern region, $87 \%$ of all new mothers were screened and $10 \%$ were followed up, half of them with 'listening visits' and the other half with a referral to psychologists or general practitioners. ${ }^{7}$ Despite these achievements, it has proved challenging to reach immigrant mothers, who are less likely to be offered counselling and less often agree to participate in screening; this group is at especially high risk of postnatal depression.

\section{Perinatal psychiatry}

Women at risk of, or suffering from, severe mental disorders require rapid access to perinatal care from specialist psychiatric units. There is a need for multidisciplinary cooperation between psychiatry and antenatal/CHS and social services, but Sweden lacks specialised perinatal psychiatric services. Ideally, the management of perinatal mental disorders is provided by experts with appropriate knowledge. For example, psychiatric units specialising in affective disorders may be able to provide prophylactic lithium treatment to women with severe affective spectrum disorders, but this necessitates collaboration with both obstetric and paediatric services. ${ }^{9}$

Access to treatment and care for women with severe mental disorders still needs considerable improvement. A study on maternal suicides in Sweden found that $47 \%$ of the 103 women who died by suicide between 1980 and 2007 had had psychiatric disorders before their pregnancy (79\% had bipolar disorder or psychosis, substance use disorders or a history of suicide attempts). But fewer than half the women's antenatal and obstetric records had taken note of that psychiatric history and in only $18 \%$ was there any record of prior suicide attempts. Although $30 \%$ had had at least one consultation for mental health problems within the antenatal care system, only $8 \%$ had received in- or out-patient psychiatric care. ${ }^{10}$

To increase access to psychiatric care during pregnancy, some obstetric and psychiatric clinics have initiated consultation-based cooperative activities. In one of these services, about $5 \%$ of pregnant women had been referred to a perinatal psychiatric liaison team during the past 10 years. Referrals originated mainly from antenatal and primary healthcare centres, but also from obstetric and specialist psychiatric services. ${ }^{11}$ A perinatal liaison team can both offer treatment and act as a gateway linking the different healthcare providers needed to manage pregnant women with severe mental disorders.

Providing equal access to healthcare for all the population in Sweden is challenging because of the low population density outside our few larger cities. During recent years, there have been efforts to improve local knowledge about perinatal psychiatric disorders through the publication of regional and national guidelines and multidisciplinary educational projects. Providing increased collaboration between psychiatric and primary healthcare providers, social services and obstetric services, such projects will be more rapidly implemented than the development of specialised perinatal psychiatric services. Mother and baby units do not yet exist in Sweden, but their formation is under discussion.

\section{Priorities for research and service development}

Our first priority is to ensure that there is rigorous assessment of the different screening protocols for risks to antenatal mental health. There should be national guidelines, based on scientific evidence, to complement the existing guidelines for screening in the postnatal period. Interventions for the prevention of postpartum depression tend to be more effective when targeting women at risk and also when offered early after delivery. ${ }^{12}$ Current projects are using machine learning and deep learning techniques to develop algorithms that will allow us to identify women at high risk of postpartum depression with greater accuracy. Further research efforts should be encouraged. ${ }^{13}$

Better organisation of perinatal mental healthcare services would improve collaboration between obstetric and psychiatric services, leading to more effective prophylaxis and treatment. ${ }^{9}$ There is a need to lower maternal suicide rates in Sweden; we should increase detection of those at risk, provide individualised planning and quick access to psychiatric care. ${ }^{10}$ Research priorities include discovering who is missing from existing screening programmes and we should plan interventions to address that problem.

\section{Conclusions}

A universal screening programme for postnatal depression is implemented within the Swedish healthcare system but it does not necessarily lead to effective therapeutic action. A structured programme to detect and treat mental health problems during antenatal care, equivalent to perinatal mental health multidisciplinary teams, needs to be developed and evaluated in all parts of the country.

\section{Author contributions}

Professor Birgitta Wickberg initiated the paper, planned and wrote all parts, except the section 'Perinatal psychiatry', which was written by Dr Margareta Blomdahl Wetterholm and Dr Marie Bendix. Professor Alkistis Skalkidou oversaw the preparation of the whole paper.

\section{References}

1 Statistics Sweden. Befolkningsstatistik: Utrikes födda i Sverige [Population Statistics: The Foreign-born Population in Sweden] SCB (www.scb.se/hitta-statistik/Sverige-i-siffror/manniskorna-isverige/utrikes-fodda). Accessed 15 May 2019. 
2 Hjern A (2012) Migration and public health in Sweden: the national public health report 2012. Chapter 13. Scandinavian Journal of Public Health, 40(suppl 9), 255-67.

3 Social Insurance Agency in Sweden [Forsakringskassan] (2016) Psykisk ohälsa. Korta analyser [Mental Illness. Short Analyses], p. 2. Forsakringskassan.

4 Howard L, Molyneaux E, Dennis CL, et al (2014) Non-psychotic mental disorders in the perinatal period. Lancet, 384, 1775-88.

5 National Collaborating Centre for Mental Health (2014) Antenatal and Postnatal Mental Health: The NICE Guideline on Clinical Management and Service Guidance. Updated Edition with notes added April 2018 (Clinical Guideline CG192]. British Psychological Society \& Royal College of Psychiatrists.

6 Swedish National Board of Health and Welfare (2010) Nationella riktlinjer för vård vid depression och ångestsyndrom [National Guidelines for Care of Depression and Anxiety Syndromes]. Swedish National Board of Health and Welfare (www.sfbup.se/vårdprogram/nationella-riktlinjer-för-vård-viddepression-och-ångetssyndrom/)

7 Centre of Excellence for Child Health Services [Kunskapscentrum Skane] (2018) Årsrapport 2017 (Annual Report 2017). Region Skane. (www.vardgivare.skane.se).
8 Shakeel N, Sletner L, Falk R, et al (2018) Prevalence of postpartum depressive symptoms in a multi-ethnic population and the role of ethnicity and integration. Journal of Affective Disorders, 241, 49-58.

9 Forsberg L, Adler M, Römer Ek I, et al (2018) Maternal mood disorders and lithium exposure in utero were not associated with poor cognitive development during childhood. Acta Paediatrica, 107, 1379-88.

10 Esscher A, Essén A, Innala E, et al (2016) Suicides during pregnancy and 1 year postpartum in Sweden, 1980-2007. British Journal of Psychiatry, 208, 462-9.

11 Blomdahl Wetterholm M, Bendix M, Pettersson K, et al (2018) A Swedish example of integrated perinatal mental health care. Lakartidningen, 115, pii: E9ES.

12 O'Connor E, Senger C, Henninger M, et al (2019) Interventions to prevent perinatal depression: evidence report and systematic review for the US Preventive Services Task Force. JAMA, 321, 588-601

13 Curry S (2019) Interventions to prevent perinatal depression: US Preventive Services Task Force, Recommendation Statement. JAMA, 321, 580-7.

\title{
THEMATIC \\ Perinatal mental health around the PAPER world: priorities for research and service development in Italy
}

\author{
Pietro Grussu, ${ }^{1} \odot$ Ilaria Lega, ${ }^{2}$ Rosa Maria Quatraro ${ }^{3}$ and Serena Donati ${ }^{2}$
}

${ }^{1}$ Family Service Unit, South Padua District, AULSS 6 Euganea Regione Veneto, National Health Service, Italy. Email: pgruss@tin.it

${ }^{2}$ National Centre for Disease Prevention and Health Promotion, Istituto Superiore di Sanità - Italian National Institute of Heath, Italy

${ }^{3}$ Hospital Psychology, Obstetrics and Gynaecology Unit, AULSS 8 Berica, Regione Veneto, Nationa Health Service, Italy

Keywords. Community mental health teams; depressive disorders; perinatal psychiatry.

First received 22 Sep 2019 Accepted 23 Oct 2019

doi:10.1192/bji.2019.31

(c) The Authors 2019. This is an Open Access article, distributed under the terms of the Creative Commons Attribution licence (http://creativecommons.org/ licenses/by/4.0/), which permits unrestricted re wse, distibution, unrestricted re-use, distribution, and reproduction in any mediu provided the original work is properly cited.
In Italy, most studies on perinatal mental health and initiatives aimed at improving the early detection and management of perinatal mental disorders have been carried out at the local level. National population-based studies are lacking. A study of pregnant women, recruited and diagnosed by a university hospital, found a $\mathbf{1 2 . 4 \%}$ prevalence of minor and major depression during pregnancy, and a prevalence of $9.6 \%$ in the postpartum period. In a population-based surveillance system, covering $77 \%$ of national births, suicide was identified to be one of the main causes of maternal death within the first year after birth, yet half of those who were known to have a high suicide risk during the postpartum period had not been referred to a mental health service. The value of recognising depressive or anxiety symptoms early, during pregnancy, has been emphasised by recent research and should be linked to multiprofessional psychosocial interventions. Since 2017, the Italian public primary care services that are dedicated to pregnancy assistance (Family Care Centres) have been tasked to provide free psychological assessment to pregnant and postpartum women. Action is now needed in order to improve access to
Italian Family Care Centres for pregnant women and to develop an integrated care model involving obstetric and mental health services.

Italy had about 60.3 million inhabitants and more than 430000 live births per year in 2018. Free comprehensive coverage is provided by the National Health Service (NHS). Responsibility for healthcare is shared by the central government and the 20 Italian regions. All citizens have access to healthcare coverage through local health units (LHUs), each of which manages a geographically defined catchment area. Treatment in public and accredited private hospitals is free of charge to the whole country.

After many years of limited interest in perinatal psychopathology, there has been growing awareness of the problem, owing to several events. First, in the early 2000s, the Edinburgh Postnatal Depression Scale (EPDS) was available in Italian translation. ${ }^{1}$ Second, a research group of experts on postpartum depression was established, coordinated by Piero Morosini at the Italian National Institute of Health. Third, the document Help to Pregnant Women and Postpartum Depression was issued by the Italian National 